\title{
Today's ecological relevance of Bergson-Einstein debate on time
}

\section{Abstract}

The reflections on the nature of time in Relativity Theory will be hinted in reference to the new bridges recently proposed by Connes and by Rovelli's perspectival approach, two major steps towards a unification of quantum, thermodynamical and relativistic times. The so-called "time of philosophers", a time of the cognizing ego, from Saint Augustin to Husserl and Bergson, is based on a different, but relevant perspective and it has been traditionally opposed to the "time of physicists". In between these two approaches, we discuss a proper time of phylogeny and ontogeny, in biology, with their own rhythms and specific irreversibility. On the one side, biological time needs to be scientifically objectivised as an invariant of the knowing subject and thus move, as in physics, from the subjective-absolute to the objective-relative (Weyl's approach, extended to time). On the other, we propose a "geometry" of life's rhythms and an "extended present" that radically differ from the prevailing spatialization of physical time that Bergson soundly criticizes. The proper irreversibility and the central, "operatorial", role of time in biology will be stressed, as nothing in biology can be understood except in the light of a temporal perspective, both evolutionary and organismal. In particular, today's eco-systemic changes bring to the limelight some disruptions of the evolutionary fine-tuning of biological rhythms and physical clocks that may be better understood by highlighting their theoretical differences as well as their environmental interactions.

\section{Introduction}

It is to the credit of Bergson's philosophy

to have pointed out forcefully this deep division between the world of mathematical concepts and the immediately experienced continuity of phenomenal time (la durée)

(Weyl 1918) 
In the history of science, when physicists faced a change of scale or observed different (or differently) phenomena, they invented a new theory. Then, the unification of the diverse theories becomes a major knowledge aim and, if achieved, a true revolution: the proposal of another theory that "brings together" unrelated or even incompatible phenomenal descriptions. Newton unified falling apples and planetary movements, totally unrelated phenomena for Galilei; Maxwell brought together magnetism, electricity and optics; Boltzmann correlated molecular movements and thermodynamic principles. Also Einstein made a major intra-theoric unification: the equivalence of gravitation and inertia. There may be more unifications, but not many, in view of their relevance and difficulty, such as the still open issue of the quantum vs. the relativistic fields.

As a bridging example towards biology, observe that the adequate scale of analysis required the development of an autonomous and mathematically rich hydrodynamics of incompressible fluids in continua, whose properties are not derivable from particle or Quantum Physics -in spite the fact that also incompressible fluids are notoriously composed by elementary particles. Major progresses are being made in the search for unity and borderline theories between these and other frames, see Chibbaro et al. (2015) for a broad survey, Longo (2016) for a review. Then, as for biology, note that there is a lot of water in an organism and that Classical as well as Quantum Electrodynamic effects contribute to cell's semi-permeability and macromolecular dynamics in cells. ${ }^{1}$ As a matter of fact, quantum and classical processes may blend, within a cell, and yield phenotypic consequences. ${ }^{2}$ As these phenomena are not inter-derivable or are even theoretically incompatible, ${ }^{3}$ none of the corresponding physical theory may allow, alone and even less in an inconsistent conjunction, to deduce the fundamental properties of organisms, from Darwin's principles for phylogenesis

\footnotetext{
Note: The permanent exchange with Maël Montévil continued the very stimulating work with Francis Bailly, on biological time in particular. Maël suggested to look at the disruption of the fine tuning of rhythms vs frequencies in pollinators and angiosperms, as seasonal plants. Pierre Martin-Dussaud made several constructive comments, as a physicist, and Ana Soto, as a biologist. Two anonymous referees as well as Alessandra Campo and Rocco Ronchi helped to improve the text, by a very close reading.

1 See Arani et al. (1995).

2 See Del Giudice et al. (1983); (1986). See Buiatti and Longo (2013) for more references.

3 With respect to classical or relativistic approaches, Quantum Mechanics is either incomplete or inconsistent, claims Einstein in EPR (Einstein et al. 1935), an incompatibility result with classical and relativistic physics. See Longo (2018a) for a comparative analysis with other "incompleteness" theorems.
} 
to our modest proposals for ontogenesis. ${ }^{4}$ A future unified theory of the fragmented physical frames, at least those witnessed in a cell, may be of major help also in biology. For the time being, it may be wise to work, following Darwin and many others, to an autonomous theorizing of biological processes, in search for a future unification, as it mostly happened in physics when facing new phenomena or observing differently old ones. Then, perhaps, it may turn out to be needed to see physics (or its mathematics in homogeneous phase spaces) as a special case of a suitable theoretical frame for biology and its heterogenesis (Longo 2020) -the mathematics for heterogenesis in Sarti et al. (2019) will be hinted below.

The necessary theoretical richness, that requires a permanent dialogue of theories, applies also to the different approaches to time, the focus of this paper. It is then surprising to observe, on one side, the technical depth of the debate on time in Relativity Theory and, on the other, the attitude of many physicists who consider the Relativistic (or Quantum, not both) Theory of Time as ... a theoretical "absolute". Claims such that "time does not exist", because "it is not present in the fundamental equations", make us think that this is too bad for the fundamental equations that, in spite of their beauty and relevance, may thus remain confined to a specific theoretical frame and phenomena. ${ }^{5}$ In particular, in no way they would help to discuss biological time, unless we invent a new unifying theory-a remote target-or at least some bridging ideas - the aim of this paper in relation to Rovelli's perspectival approach to time. In order to construct a bridge, though, one needs the two sides of the ditch, in particular an autonomous theory of biological time. On the one hand, we refer to Rovelli's approach to time in physics that is based on an analysis of the relativizing choice of the thermal coarse graining. ${ }^{6}$ On the other, we develop previous work on biological time and rhythms in collaboration with Bailly and Montévil (see references) and frame it by the notions of characteristic time and time scales for biological functions, as defined by the "closure of constraints" in Montévil and Mossio (2015).

We will thus not present a historical account of the Einstein-Bergson debate, but refer to some its developments in order to propose a perspectival epistemology of time in the sciences of nature and hint to its relevance in the current ecosystemic crisis, partly due to a poor understanding (or little care) of the physical

\footnotetext{
4 See Soto et al. (2016).

5 The general form of a quantum dynamics of variables, with no time parameter, has been given by Wheeler and De Witt (see Rovelli 2008). Wheeler-DeWitt equation is a variant of Schrödinger equation in a diffeomorphism invariant (relativistic) context, a tentative formal bridge between the two theories.
}

6 See Rovelli (2015). 
singularity and autonomy of life. A pertinent theory of biological time may also contribute to the philosophical analysis of pre-conscious or conscious (intentional) time (the "time of philosophers"): biological rhythms may induce a primary form of protension, as pre-conscious expectation; the mathematical "gluing" of retension (pre-conscious memory) and protension will point towards a simple formalization of the subjective experience of the continuity of phenomenal time. Both analyses may help to objectivize some aspects of Bergson's notion of durée as well as its relation to continua and thus to recompose the deep division stressed by Weyl (in epigraph, above).

\section{The Thermal Time Hypothesis and the Regulating Objectivity of Physical Time}

All a priori statements in physics have their origin in symmetry

(Weyl 1952)

In the commonly used mathematics of modern physics, the Cantorian one dimensional continuum as a line of points with no jumps nor lacunae, identically describes and entirely displays under our eyes both space and time. Thus, Weyl's unsatisfaction in epigraph to the first section: there is no way to isolate the phenomenal/perceived time of the present as a Cantorian point; the mathematical continuum does not allow a privileged interval of measurement or of conscious access to time. Bergson's durée instead is an incompressible, indivisible interval, where consciousness of the present is possible as it is coextensive to memory of the past and expectation of the future (Bergson 1947, 195), an approach that permeates philosophy, from Saint Augustine to Husserl. In this perspective, future cannot be displayed as a line given in space, even less if made out of Cantorian points.

Moreover, Weyl, the author of Space, Time, Matter, the 1919 founding book for the mathematics of Relativity, is well aware that (General) Relativity Theory $((\mathrm{G}) \mathrm{RT})$ moved the description of time even further away from the cognitive/experienced time, since it allows no preferred independent time variable: spacetime may be understood as a unique "block" subject to the same transformations; up to minor differences, space and time yield the same invariant properties under these transformations (diffeomorphism invariance or general covariance). In this sense, GRT definitely spatialized time and erased its specificity; in partic- 
ular, its orientation, as a flow from past to future, disappears. ${ }^{7}$ Finally, and this is mathematically crucial, one cannot describe a relativistic dynamic as an evolution depending on a pre-given time parameter. ${ }^{8}$

However, as for the choice and the orientation (the "arrow") of the time parameter in physics, Connes and Rovelli (1994) made major contributions by proposing a triangular relation between the analyses of time in quantum (QM), thermodynamical (TD) and relativistic frames (GRT).

In short, recall first that measurement in QM does not commute: if the speed of a particle is measured first and the position later one obtains a different result from a measurement done in the inverse time order. This sets an orientation of time. In TD, instead, the time orientation is given by increasing entropy, which may be expressed as a move from a fine-grained to a coarser grained access to phenomena. That is, from a more to a less detailed description of its microscopic behaviour, or a description in which some of these fine details have been smoothed over. ${ }^{9}$ In a given system, a given temperature, typically, may yield a coarse-grained, macroscopic, representation of the particles in aggregate. Energy transformations, as they tend to reduce differences in temperatures, increase entropy and lead to a coarser graining of the intended system. Thus, measurement or the choice of a coarse graining fix a dimension and a direction of time, in QM and TD, respectively, as argued next.

By building on classical results by Gelfand, which allow to reconstruct (Riemannian) geometry from commutative algebras, Connes (1994) has been reconstructing, for many years, the geometry of QM in terms of non-commutative algebras. Then, he unified, mathematically, the orientation of time due to quantum measurement and the thermodynamic understanding of the arrow of time in terms of entropy. ${ }^{10}$ In short, the arrow of time defined by quantum non-commutativity and the one determined by macroscopic states (or coarse

7 The view points within RT and its philosophy, beginning with Einstein late reflections on time, differ, see (Bouton and Huneman 2018) on this debate. Under all interpretations though, "you will never derive the idea of a temporal flow from Minkowski's schema” (Bergson 1965, 63). 8 See Connes and Rovelli (1994). "The general form of a mechanical theory that describes the evolutions of variables with respect to each other is given by a phase space and a constraint $\mathrm{C}$. The relations between the variables are given by the orbits generated by $\mathrm{C}$ in the subspace $\mathrm{C}=0$. The parametrization of these orbits has no physical meaning” (Rovelli 2018a, 132ff67). As already hinted, the general form of a quantum dynamics of variables, with no time parameter, is Wheeler-De Witt equation.

9 Entropy, in short, is the logarithm of the number of ways that the insides (of the intended phase space) can be re-arranged, so that from the outside it looks the same. See Feynman (1964) for an excellent introduction.

10 See Connes and Rovelli (1994). 
graining) in TD may be described by the same algebraic frame -the non-commutative von Neumann algebras'. These define an a-symmetric "flow", which may be interpreted as an oriented time in either theory.

Now, Rovelli's Thermal Time Hypothesis refers precisely to the statistical state, or the statistical distribution given by the chosen coarse graining, as determining physical time and its direction (i.e., moving towards a coarser graining). ${ }^{11}$ The coarse graining or blurring is due to the fact that we are ignorant of the microscopic details of the observed process. The time of physics is, ultimately, the expression of our ignorance of the world, or of the limitations of measurement, in particular. Note that in both QM and TD, measurement or quantitative access to phenomena, as indetermination or coarse graining respectively, are crucial. In this double sense of blurring then one may understand Rovelli's "time is ignorance".

However, the interaction of different subsystems may suffice to fix a "statistical state that determines which variable is physical time", with no need of an "a priori hypothetical 'flow' that drives the system to a preferred statistical state" (Rovelli 2011, 8). That is, measurement may be replaced by the interaction of different systems, in principle sub-systems of a "larger" system. After all, a measurement instrument in QM is a classical (macro) system that, by a 'friction' or coupling with micro-phenomena, co-constitute the observable properties and states of the measured particle. Similarly, as for the choice of the coarse graining in TD. Note that different choices of macroscopic observables and coarse graining, in the same process, may lead to opposing time directions. ${ }^{12}$ In reality, this choice is not arbitrary as it depends on the specific coupling between the intended systems. But which subsystems may force an (oriented) time parameter in the interaction with other subsystems? In the next section we will discuss a fundamental one: a living cell.

In other words, time is perspectival, as Rovelli calls it, as it is relative to a specific access or coupling friction that fixes either the order of measurement of quantum observables or the coarse graining in the analysis of thermodynamic processes. Time is as relative as speed, since it similarly depends on the reference system, if we just broaden the notion of reference system to the choice of the order of quantum measurement or of the coarse graining. The analysis of the transformations and their invariants with respect to reference systems at uniform relative speed, Galilei's relativity, made the analysis of motion objective and started modern science: the laws of motion are invariant w.r. to Galilei's transfor-

11 See Rovelli (1993); (2008).

12 A simple visual example is given in Rovelli (2015). 
mations. A relativizing understanding of oriented time, by considering also the access to phenomena as part of the choice of reference systems, may give the proper invariance properties and the corresponding transformations, and thus construct a new objectivity for the "order of time". By an instrumental use of a Kantian terminology, we may then say that objectivized (oriented and measurable) time acquires a regulating role in physics: it contributes to the intelligibility of certain phenomena, by ordering them. Typically, it is mathematized as a totally ordered parameter in thermodynamic diffusions and entropy. Yet, it is not constitutive, in the sense we will propose in biology: the underlying particles' trajectories make the processes intelligible with no need of an oriented time, which is an a posteriori result of a statistics. Note, instead, that conservation properties (of energy, momentum ...), which may be described as symmetries, by Noether's Theorems, ${ }^{13}$ are constitutive and posited as a priori in physics, as Weyl would say. That is, they participate to the co-constructed existence of physical objects and allow the objectivity of mathematized laws and deductions. ${ }^{14}$ On the contrary, the orientation and the origin of time are major symmetry breakings - and no "a-symmetric flow" may be a physical a priori, following Einstein, Weyl, Rovelli. And time has lost its transcendental, a priori status in physics.

However, in spite of the common linear-cantorian representation, the epistemology of time is a controversial one in physics, even in a regulating, non-constitutive role. From the relativistic orthodoxy on the fundamental non-existence of time, from McTaggart (1908) to Callender (2017), and the weak (purely relational) and reversible forms of time in GRT in Van Frassen (1985), to the proper irreversibility of thermodynamical time in Nicolis and Prigogine (1977). Now, also relativistic time needs to be revised in view of the novelties in Cosmology. Cosmology is a "historical science" and it should deal with an irreversible time with an origin, the Big Bang, in the view of many cosmologists; it then presents major challenges for timeless theories. ${ }^{15}$ Thus, while thermodynamics analyses

13 See Kosman and Schwarback (2010); Longo and Montévil (2014).

14 For example, an apple falls, a planet moves for "symmetry reasons", since GRT unifies gravitation and inertia in Riemann's manifolds and inertia is a conservation property. Following Weyl, gauge symmetries (Yang and Mills 1954) geometrize also non-gravitational interactions. And a physicist may say today: this particle must exist for symmetry reasons -and then he/ she knows what and where to measure. Moreover, equations, from Newton to Navier-Stokes, Einstein and Schrödinger, are written and solved in a phase space, a priori given by the intended theory, a fundamental invariance or symmetry of the theory.

15 In the strict relativistic view, intersecting cones of the future with different apex points, in Minkoswki's representation, must contain identical events. These events must then be fully and a priori determined, as no coordination is possible between remote apex points. Thus, 
irreversible processes where time assumes a key regulating role, we are far from a unified understanding of GRT, quantum and statistical mechanics or thermodynamics. Along the lines hinted above, a possible broadening of GRT is being proposed under the form of a "general relativistic quantum statistical mechanics". ${ }^{16}$ In this perspectival approach, the relativizing choice of the time dimension and its direction, by a coarse graining, regulates knowledge construction by an observer. Yet, it may also be analysed in terms of interacting subsystems, as mentioned above: the interaction fixes the coarse graining. A major opening towards our approach in biology.

\title{
3 The Intrinsic Objectivity of Biological Time
}

\author{
In physics, a lowered energy state is not necessarily disorder, \\ because it simply results in the identical molecule with a lowered energy state. \\ The fact that such a molecule might be biologically inactive \\ may not concern the physicist, but it definitely does concern the biologist
}

(Hayflick 2007).

An organism, a cell, fixes the thermodynamic coarse graining at the molecular scale: typically, it is viable only in a certain interval of temperature, a measure of energy transformation, thus of entropy production. More generally, the biological function of any component of an organism forces its entropic level, within an interval of viability, according to the organism and its context. The dimension and the direction of thermal time is then fixed. We call intrinsic or constitutive the approach to time in biology hinted in the cursory review below, following Bailly et al. (2011), Longo and Montévil (2014) and Longo (2017).

\subsection{Biological Rhythms vs. Physical Frequencies}

The analysis of time in multicellular organisms requires first a key distinction between physical and biological clocks. The spinning Earth, the relative movements of the Moon and the Sun have the dimension of (the inverse of) time (a frequen-

not only the space of possibilities (the phase space) is given a priori, but even the potentialities are actual according to this interpretation, see the debate in Bouton and Huneman (2018). 16 See Chirco et al. (2016); Rovelli and Vidotto (2018); Dorato (2016) for a philosophical reflection on Rovelli's Relational Quantum Mechanics. 
cy) and set fundamental physical clocks for life: days, months, seasons. ${ }^{17}$ On top of them, many organisms constructed their autonomous rhythms, such as heartbeats and respirations. All mammals, say, have the same number of total heartbeats and respirations, on average $1.2 \times 10^{9}$ and $0.8 \times 10^{9}$, respectively, in their lifespan, a major biological invariant in wild species. So, a wild mouse or elephant, whose lifespans are of about 2 and 80 years, respectively (on average and varying with the species), have about a 40:1 ratio of heart frequency per minute (up to about 600 beats per minute for a mouse and 15 beats for an elephant). ${ }^{18} \mathrm{Sim}$ ilarly as for respiration frequencies, once one scales the number $0.8 \times 10^{9}$ above to a species' average lifespan. These numbers must then be understood in terms of "pure numbers": they have no physical dimension, but scale to a frequency, that is to the dimension of (the inverse of) time, once they are referred to a life span -which has an average allometric dependence on the 1/4th power of the biomass in wild animals. ${ }^{19}$ Thus, biological rhythms do not depend on the physical clocks mentioned above, but are tuned to them, like during night sleep or hibernation. Some frequencies and rhythms are very closely tuned, such as the day/ night (circadian) frequency that forces a biological rhythm: endocrine activities (melatonin production among others) internalize, as a circadian rhythm, the external circadian frequency. In case of jet-lag, we need a few days to re-adjust the internal endocrine circadian rhythm to the day/night frequency.

This distinction, rhythms vs. frequencies in biology, is crucial, yet it is rarely formalized. ${ }^{20}$ In short, evolution set organismal internal clocks, such as heartbeats. ${ }^{21}$ These rhythms are or became independent from physical frequencies and constitute major biological invariants, as hinted above. A simple geometric representation of both may be obtained by adding to the oriented dimension of thermodynamical time a second compactified dimension (a circle), in the style of

17 A physical clock sets a frequency (dimension: inverse of time) by an oscillation, a circular movement, an irradiation. Thus, it fixes the dimension of time and a metrics on it: "time is movement that may be counted", beautifully says Aristotle.

18 Note that Galilei used his own heartbeats in order to measure the frequency of a pendulum, the lamplight in the cathedral of Pisa, and this leads to the invention of clocks regulated by pendula. Then, he used time as a parameter to describe falling bodies, a revolutionary step in physics.

19 See Günther and Morgado (2005); Longo and Montévil (2014) for details and references. 20 We tried in Bailly et al. (2011); Longo and Montévil (2014).

21 The setting up of heartbeats is not the result of a programmed oscillator, but of a systemic property: at a critical transition during embryogenesis, interacting embryonic heart membranes, ion's flows, cells' microtubules and neural oscillations resonate and set the rhythm. This gradually correlates to the entire body physiology and even regulates pertinent gene expressions. See Noble (2006); (2012). 
Kaluza-Klein theory in physics as for space. ${ }^{22}$ This yields a "cylinder", as a twodimensional manifold or a geometric schema for biological time, unrelated to space. Rhythms are then described as spirals along the cylinder, an effective diagram for the interplay of irreversible physical processes (linear time dimension) and internal clocks (spiraling along the cylinder). ${ }^{23}$ Our schematic cylinders allow to represent the independence and tuning of the two dimensions of biological time and apply to each individual organism, by a locality of time representation that resembles Rovelli's disordered, localized time-cones in Rovelli (2018, Ch. 3). However, in contrast to Rovelli's space-time cones, the inter-organismal fine tuning of rhythms and frequencies is at the core of our ecosystemic perspective on time, as hinted next.

Organisms use also accumulators to measure physical time. Cicadas, which live 13 or 17 years underground before hatching, use an accumulator of the sugar absorbed from the roots of trees in order to emerge with the frequency set along evolution ${ }^{24}$-the surprising prime numbers are probably selected since they cannot be divided by reproduction times of predators. In their dance, bees communicate to the others in the beehive the flying time to pollens. That time is measured by internal accumulators and rhythms, which are used also to estimate foraging time: even when the circadian frequency is experimentally disabled, foraging honeybees follow the correct interval timing, suggesting that the systems are independent. ${ }^{25}$ With the current climate change, as angiosperms follow the seasonal temperature, an increasing temperature may force early blossoming, so that pollinators may reach different flower species with wrong timings. ${ }^{26}$ The pollinators/flowers evolutionary fine tuning of internal rhythms vs. external frequencies, in foraging and pollination, is a very relevant phenomenon for many food chains and may then be disrupted. A mechanistic view of time or of nature does not help in seeing these ecosystemic disruptions. ${ }^{27}$

22 See Wesson (1999).

23 See the figures in Bailly et al. (2011); Longo and Montévil (2014), https://www.di.ens.fr/users/ longo/download.html

24 See Williams and Simon (1995).

25 See Foster and Kreitzman (2004).

26 See Memmott et al. (2007).

27 Many more cases of relevant synchronicity disruptions may be found in the literature; yet another major one is the ongoing de-sinchronization of reproductive spawning in corals (Shlesinger and Loya 2019). For a synthetic analysis of ecosystemic disruptions, including chronobiological ones (see Montévil 2020a). 
Rhythms and frequencies fix durées: typically, the time in between two iterations of a rhythm. Accumulators add further measurable time intervals. ${ }^{28}$ An ecosystem is a tissue of correlated and recorded durées, as Bergson would put it. $^{29}$ The changes in this tissue or heterochrony, as altered characteristic times in ontogeny, contribute to evolutionary changes. For example, in the threespined stickleback (Gasterosteus aculeatus), heterochrony in the expression of adaptive traits contributed to speciation in a limnetic and a bentic form. Their

ancestral population occupies both of the habitats observed in the descendent species pairs and exhibits both phenotypes at different times during its life cycle, a pattern that suggests that the different recurrent forms may have originated not by parallel evolution but by altered timing (heterochrony) in the expression of those traits (West-Eberhard 2005, 6546). ${ }^{30}$

Time scales and characteristic times are then crucial notions in biology. The "closure of constraints" describes biological functions as part of a "mutual dependence between a set of constituents which could not exist in isolation, and which maintain each other through their interactions" (Montévil and Mossio 2015, 180). Each functional closure applies at a precise time scale and for a characteristic time. Functions then define biological rhythms, such as metabolic, endocrine and cardiac rhythms: from enzymes, which have a time scale and a characteristic time related to their catalytic activity and to the (un-)binding to a substrate in a reaction, to major organismal systems, such as the vascular system, all have a proper time scale and a characteristic time. ${ }^{31}$ During embryogenesis, the increasing levels of functional nesting and interactions in the forming organism can be analysed in terms of a complexifying closure of constraints. This provides a properly biological and measurable "coarse graining" of organization: an increasingly finer (more complex) organization sets the strictly irreversible and biological arrow of time of growing functionalities in an organism, while producing new autonomous rhythms. ${ }^{32}$ If your pet theory of organisms allows to conceive the

28 "Wherever anything lives, there is, open somewhere, a register in which time is being inscribed” (Bergson 1922, 17).

29 See During (2009); Ronchi and Leoni (2007).

30 See Huneman (2018) for more aspects of timing and time scales in micro and macro-evolution.

31 The ions' flow of an action potential in neurons requires about a millisecond to travel a few nanometers, which is much more than the usual time scale in physics for these molecular processes (Lesne 2018). The biological functions and contexts impose their own scales and characteristic time.

32 The anatomical complexity of an organism can be measured Bailly and Longo 2009; Longo and Montévil 2014. We called "anti-entropy" this new observable whose space geometry and di- 
formed baby to move backwards to the unorganized morula or the zygote, you should better forget it: it has no biological meaning - only death suddenly destroys biological organization (or, locally, illness). Time irreversibility of biological dynamics is not "just a matter of probability", as physicists soundly say in thermodynamics, but of an irreversible construction of organization and its rhythms, as functional "closure of constraints". In this sense, the measurable time of biological setting up and maintenance/renewal of organization is a new observable time, which we theoretically distinguish from thermal time-similarly as one can distinguish potential and kinetic energy in the dimension of energy. ${ }^{33}$ More will be said below in reference to evolution.

Note that organismal rhythms, such as the cardiac rhythm, force a form of protension: the preconscious expectation of the iteration of the rhythm - such as heartbeats. ${ }^{34}$ The correlation of frequencies and retension/protension may be witness even in amoebas, which anticipate periodic events. ${ }^{35}$ In humans, musical notes and rhythms require a join of protension and retension, in a least time durée, in order to produce musical sense-similarly as for the retension and protension required to understand language. This "gluing" (a mathematical notion) of retension and protension in an interval is a fundamental durée in all forms of perception and, eventually, in consciousness of time -a simple mathematical description of this phenomenon is given in Bailly et al. (2011, sect 5.4), Longo and Montévil (2011a). In our view, the "continuity" that we attribute to a trajectory (of a prey, a ball ...) results from glueing retension and protension of it, including the protensive eye jerks and brain's associated re-organization described in Berthoz (2000). ${ }^{36}$

mensions matter (in contrast to negentropy as one-dimensional information). Anti-entropy adds, and does not opposes, to entropy, produced also by its very setting up, as in all irreversible processes -each cell reproduction increases anti-entropy while producing entropy, by energy transformations and by the slight disorder of the a-symmetric division. In short, philo- and onto-genesis simultaneously produce entropy and quantifiable, three dimensional and metric organization (anti-entropy). Montévil and Mossio (2015) further specified it by adding functional closure, a key notion in biology.

33 Sarti el al. 2018 consistently propose to introduce another dimension for this new observable time. This is fascinating and may require the invention of a new pertinent dimensional constant to relate the two forms of time, such as Boltzmann k in TD, say, a non-obvious step.

34 See Noble (2006).

35 See Saigusa et al. (2008).

36 A frog would never claim that movement is continuous: it sees it by scattered snapshots. We, large vertebrates, follow and precede moving preys by continuous eye jerks and, very recently, we invented continuous background spaces and their mathematics (Longo 2020). The Cantorian, a posteriori reconstruction of phenomenal continua by dimensionless points, justifies Weyl's dissatisfaction: it is very powerful and rigorous, it founds the point-wise time instants of the 
Finally, organisms continually re-construct themselves, by somatic cell reproduction. Each of these reproductions has the characteristic of a critical transition: a re-organization of internal and external symmetries-from one to two cells; the formation of new coherence structures-the tissue matrix, re-constructed collagen fibers etc. In an immensely more complex way, this resembles to a para/ferro-magnetic critical transition, the formation of a snowflake. ${ }^{37}$ In physics, critical transitions are formalized as a point-wise process by the divergence of (the derivatives of) some function of the dynamics on one point of the pertinent parameter. In biology instead, the durée of these transitions is crucial as it internally contains several, nested, critical transitions (e.g., DNA split, proteome's reorganization ...) that make no sense in isolation. Moreover, a multicellular organism undergoes thousands of cell reproductions, thus of these nested, extended transitions, in short time intervals. The notion of "extended criticality" in Bailly and Longo (2011); Longo and Montévil (2011b), unknown to mathematical physics, may help to grasp the peculiarities of this continual reorganization which is proper to life; it may be viewed as a topologically dense interval of critical transitions, in a non-cantorian continuum. And, at the proper scale, extended criticality yields incompressible, non-divisible durées in the pertinent phase space, the extension of the interval of criticality. ${ }^{38}$

In summary, the analysis of biological rhythms in terms of closure of constraints provides an objectivizing-relative theoretical frame for Bergsonian durées as pertinent and measurable characteristic times and time intervals, at different time scales. Similarly, perception should be analysed in incompressible intervals of time or by glueing retension and protension. Moreover, critical transitions are omnipresent but extended to time intervals. All these durées, in order to be understood in their functionality, cannot be arbitrarily compressed nor divided, unlike a Cantorian time-segment. They set the time of Darwin's “correlated variations" and of interacting causality in all scales, as described by Noble's "Biological Relativity" (Noble 2012). We also hinted to the role of variable durées in phylogenetic heterochrony; more should be said as for changing time interactions in embryogenesis. ${ }^{39}$ In short, the analysis of the network of functional $d u$ -

XVIII century differential calculus, but misses the a priori of perception and of its biological timing, as durées. Better can be done by more modern mathematics (see the footnotes in §5). 37 See Binney et al. (1992).

38 "The thing and the state are only artificially taken snapshots of the transition; and this transition, all that is naturally experienced, is duration itself" (Bergson 1965, 44). For Bergson the concept of duration encompasses both the idea of passage and of conservation.

39 See Raff and Wray (1989). 
rées, at all levels of organization, and of their changes in organisms and ecosystems is a core biological investigation.

Recall then a major epistemic lesson of Einstein's GRT:

The geometry of relativistic spaces is a tissue of interactions: when deforming these interactions, the tissue and its geometry change; conversely, a deformation of the geometry changes the interactions, their tissue.

The constitutive role of time in biology and its deformations may be analogously synthesized:

The time of an ecosystem is a tissue of interacting rhythms and frequencies: when deforming these interactions or their tissue, rhythms, frequencies and their tuning change; conversely, a deformation of rhythms or frequencies and of their tuning modifies the tissue, the time of the ecosystem.

Of course, a multicellular organism is also ecosystem, often inhabited by more bacteria than somatic cells. Yet, the converse does not need to hold. In particular, the resilience to time and space (metric) deformations of the tissue of interactions may be incommensurably higher in an ecosystem than in an organism. In biological ecosystems, of either type, the notion of pathology or of disruption are perfectly sound and relevant, in particular in reference to the functional finetuning of rhythms and frequencies, while they are not in physics. The current ecosystemic crisis requires a close attention also to the changes in the evolutionary network of recorded durées, as defined by Bergson.

\subsection{The Time of Evolution}

\section{the present moment of a living body does not find its explanation in the moment immediately before [...] all the past of the organism must be added to that moment, its heredity -in fact, the whole of a very long history. \\ (Bergson 1922)}

Another distinction is required as for the time of phylogeny and ontogeny. The phase spaces (pertinent observables and parameters) of physical theories may differ, but they are given a priori by each theory. ${ }^{40}$ From Aristotle to Newton, Ein-

40 It is the theory that fixes the observables (Einstein), which, in a given physical theory, are fixed or may at most statistically change in a pre-given list. See Disertori et al. (2015); Sethna (2006). 
stein and Schrödinger, the actual is already in potentia, in the space of all possible paths - possibly a (Cartesian) phase spaces or an infinite dimensional (Hilbert's) spaces of quantum probability's amplitudes (Schrödinger). Or, the bifurcation (ontologically) precedes the fluctuation or perturbation that induces one path or the other. ${ }^{41}$ In a Darwinian perspective, instead, the pertinent observables and pertinent parameters, thus the space of possible phenotypes and organisms, are produced during and by evolution. "The origin of a new direction of adaptive evolution starts with a population of variably responsive, developmentally plastic organisms" (West-Eberhard 2005, 6544). Variability is co-extensive with life (Darwin's first principle) and fluctuations, within organisms and organisms-ecosystem, co-constitute bifurcations. ${ }^{42}$ In other words, the phase space of evolution is produced by evolution itself, and this is inconceivable in existing mathematical physics. ${ }^{43}$ In biology, thus, on one side time is ignorance not only of what will happen, in a pre-given space of all possible "trajectories", but even of what may happen. On the other, time is the construction of new spaces of possibilities, as evolution is heterogenesis not just morphogenesis in homogeneous spaces (see below), a major mathematical challenge. ${ }^{44}$

Moreover, rare events (hopeful monsters, allopatric speciation ...) crucially contribute to phylogeny, ${ }^{45}$ in a very different way from the rare "large fluctuations" that importantly, but rarely influence trajectories in physics. ${ }^{46}$ As a matter of fact, any phylogenetic path, or most of its bifurcations and changes, are marked by and result from rare events. ${ }^{47}$

41 As observed by Thom in Thom (1990).

42 See Longo et al. (2012); Longo (2017).

43 Darwin, in a marvelous page on variation and the production of new phenotypes in evolution (Origin, ch. 5), stresses the extreme sensitivity of organisms, their contexts and their interactions to minor or non-detectable changes. Thom insightfully sees the relevance of this issue and, consistently with mathematical physics, observes that it "affects, very seriously, the scientific nature of Darwin's Theory of Evolution” (Thom 1990, 271). Great thinkers are at least aware of their a priori (and metaphysics: Thom's firm mathematical Platonism) -also when they are wrong. We only need new mathematics for this, perhaps the mathematical heterogenesis hinted in Sarti et al. (2019), see below.

44 In philosophy, Bergson opened the way to this perspective, see Bergson (1922); (1946).

45 See Longo (2017).

46 See Vulpiani et al. (2014).

47 See Gould (2002). One of the challenges of today's cosmology, which aims at a historical theory, is that both the observables and the fundamental physical constants may be considered as varying (Uzan 2011). Perhaps, some inspiration from theorizing in evolution, since Darwin, may help cosmologists, such as the focus on changing phase spaces and rare events. Economists, as they also work at a historical theory, have been already inspired from our approach (Koppl et al. 2015). 
This approach requires a further distinction. Hurricanes, flames ... and all far from equilibrium physical processes are described by an irreversible processual time, in a given phase space. They are all of the same "type", in a robust mathematical sense, since four billions years on Earth and a sound theory in a pregiven phase space, and its mathematics, effectively describes them. Life instead somewhat changed since its origin. It has a historical time, specified by changing phase spaces and by rare events. Moreover, traces of its past shape the present and the future, very differently from path dependence in physics. ${ }^{48}$ This re-use of the past may be analysed in terms of, for example, but not only:

- Gould's exaptation (adaptation ex-post: the new use of an old phenotype), degeneracy ${ }^{49}$ and overloading ${ }^{50}$ of organs and functions;

- Degenerate and multiple use of a segment of DNA by (de-)methylation, alternative splicing or overlapping genes; ${ }^{51}$

- Activation of cryptic mutations etc. ${ }^{52}$

Intelligibility thus depends also on knowledge of the past and thus, possibly, on diachronic measurement. That is, a biological function, an organism, a species are understood in terms of their history: the structure and function of brain, lungs, ... the absurd connectivity in vertebrates eyes (as pointed out by Helmholtz), if compared to the octopus's homologous one, or ... "what is a mouse" 53 can only be understood in phylo-ontogenetic terms, that is by an analysis of their constitutive history, possibly by accessing to and measuring common ancestors. ${ }^{54}$ The need for synchronic and diachronic measurement as well poses major challenges in biology: the specificity and historicity of each individual is a major theoretical issue and massively affects experimental reproducibility. ${ }^{55}$ In a Bergsonian perspective, organisms may be only understood within a life flow, their ever changing evolution. ${ }^{56}$

As mentioned above, the historical time of onto-phylogenesis may be better described by a new observable (or an extra mathematical time-dimension, see the footnote). Following this approach, in Bailly and Longo (2009), we used a

\footnotetext{
48 See Longo (2017).

49 See Edelman and Gally (2001).

50 See Longo (2017).

51 See Pavesi et al. (2018).

52 See Paaby and Rockmann (2014); Longo (2017) for more.

53 See Montévil (2019).

54 See West-Eberhard (2003); Lecointre and Le Guyader (2017).

55 See Montévil (2019).

56 See Bergson (1922).
} 
diffusion equation in order to formalize a remark in Gould (1996), concerning the (largely random) increasing phenotypic complexity in evolution. Intuitively, more "complex" organisms, in the sense of a notion that we formally define and measure (anti-entropy, see notes sect. 4), may construct/occupy new niches -thus they have more chances to fit. ${ }^{57}$ The equation, with real coefficients, is analogous to Schrödinger's wave equation in QM, which may also be understood as a diffusion equationin our case, it yields an asymmetric diffusion of bio-mass over “complexity”. Dually to Schrödinger's equation, and for good mathematical reasons, time turns out to be an "operator" and energy (or mass) a parameter, in agreement with the role of energy (or mass) as a parameter in allometric equations in biology. ${ }^{58}$ It is still hard to fully grasp the biological meaning of such a duality, yet, if our analysis is correct, it seems to stress, by a new mathematical frame, the constitutive role of time transformations, whose epistemic status becomes then similar to conservation laws for energy or momentum in physics.

\section{Comparing Theories of Time}

In physics, from Aristotle's "time is movement that is counted" to Einstein spacelocalized clocks, it is commonly understood that "time is what is measured by clocks". ${ }^{59}$ This parallels a common physico-mathematical definition of randomness: randomness is what is measured by probability (from Laplace to Kolmogoroff, in the 1930's) ${ }^{60}$ Joining the two, as for an issue we already hinted: time reversibility is just a matter of probability. ${ }^{61}$ These views, that may be perfectly sound in physics, are largely inadequate in biology.

First, organisms construct their own time as internal rhythms, which, at least in mammals, scale to bio-mass, as mentioned above. Biological rhythms do not just measure, but engender the time of organisms, a complex organismal and ecosystemic tissue constructed in evolution and embryogenesis, in relation to,

57 Anti-entropy is a different observable from negentropy: it is generated while producing entropy (Bailly and Longo 2009) and it measures phenotypic complexity as a dimensional/geometric notion, depending on fractal dimensions in organs, size of networks, such as the neural network, number of tissue differentiations. This is in contrast to negentropy as information, which is one-dimensional and dematerialized (independent from the "hardware").

58 See Gould (1966); Peters (1983); Longo and Montévil (2014).

59 See the papers on physical time in Bouton and Huneman (2019).

60 See Mugur-Schachter and Longo (2014).

61 See also the Einstein-Ritz debate in: Frisch and Pietsch (2016). In Duration and Simultaneity, Bergson argues that "the measurement of a thing is, in the eyes of the physicist, that very thing" (Bergson 1965, 159). 
but differing from physical frequencies (clocks). A possible analysis requires some more geometry than just a time-line and the counting of a frequency on it, as we hinted in §.3, in reference to the breaking of the evolutionary fine tuning of biological rhythms and physical frequencies. Stressing the difference as well as the interactions provides an understanding some of the ongoing ecosystemic changes, which can then be measured. Yet, the conceptual determination may precede measurement; for example, the operatorial role of time quoted above specifies our perspective by some mathematics, in spite of the difficulties, or impossibility, in pre-defining and measuring changing spaces of possibilities (the possible phenotypes and organisms) and rare events. More in the next item.

Second, randomness mathematically differs in classical and quantum frames, as it yields different probabilities (e.g., the violation of Bell inequalities). ${ }^{62}$ In general, randomness is unpredictability in the intended theory. ${ }^{63}$ Since, in physical theories, phase spaces are generally pre-given, probability, as a measure of randomness (Lebesgue's measure, typically), may be a priori fixed by the observer and soundly defines randomness. ${ }^{64}$ This is not so in evolutionary dynamics where the very space of possibilities is not pre-given -it does not precede the dynamics. Thus, no probability measure can be given on this "space". So, unpredictability (randomness) moves from a value within a space of pre-given observables to the very set of possible observables and cannot be measured..$^{65}$

Third, as for time reversibility, does one refer to time or to the observed process as reversible? In short, a process may be considered "reversible in time", when it is parametrized over time, $\mathrm{t}$, and setting - $\mathrm{t}$ yields a physically conceivable/possible process. Typically, Newtonian mechanics and all dynamics where the time parameter $t$ appears as a $t^{2}$, thus inversing $t$ in $-t$ poses no problem (the orbits of planets can be very well be conceived to go in the opposite direction). ${ }^{66}$ But also the diffusion of a gas can be thought as time reversible: in the atomistic perspective, since Boltzmann, the inversion of the trajectories of gaz particles is conceivable, it is just a statistical matter with very low probabilities. In some cases, the thermodynamical process (mixing gazes, say) may be reversed

62 See Aspect et al. (1982)

63 See Calude and Longo (2016).

64 However, both Poincarés analysis (1892) and the standard interpretation of QM provide an epistemic interpretation of classical and quantum randomness, respectively, which conceptually precedes probabilities. They may be both asymptotically related to a strong form of undecidability, Martin-Löf randomness (Calude and Longo 2016).

65 See Longo (2017).

66 See Gayon and Montévil (2019) for a detailed discussion. 
by some energy (a centrifuge), with no inversion of the time parameter. As mentioned above, neither chance with low probabilities nor a centrifuge would help to reverse aging nor embryogenesis, from an old man to a baby to a zygote.

In Longo and Montévil (2017), we show that in existing physical theories, the following events are invariantly correlated: a symmetry breaking, a random event and the (local-processual) irreversibility of time (in short, think to classical bifurcations, to the projection of the QM state function, to thermal diffusion ...). A remarkable mathematical unity of physics. This correlation holds also in our approach to the proper irreversible time of biology, in evolution in particular. Yet, the fundamental symmetry that is also broken by the time flow is the conservation of the phase space, i.e., by the changes of the space of possible phenotypes. As already mentioned, this yields a non-measurable form of randomness - yet a very close approach to ours may be already given a mathematical representation. ${ }^{67}$

As for the Bergson-Einstein debate, an irreversible and universal "becoming" is at the core of Bergson's philosophy of nature. It is not being that becomes, but becoming is being: ${ }^{68}$ life undergoes a permanent change not only in time but "enacted" by time. Can this be mathematically specified as a view of time as a (differential) operator, as mentioned above? Indeed, an organism is a becoming: if somatic cells stop reproducing, the organism is dead, it is no more. Species can only become and change: there is no way to stabilize them, not even in a stable environment, observes Darwin. Reproduction with modification is his first principle for species' evolution and our default state for cells also within an organism. ${ }^{69}$ Note though that also physics, QM at least, is moving beyond classical falling stones and relativistic block-universes: "The best language for describing the universe remains a language of happening and becoming, not a language of being. Even more so when we fold quantum theory in. Loop Quantum Gravity (LQG) describes reality in terms of processes" (Rovelli 2018b, 4). Yet, LQG has no preferred time variable and its becoming is a matter of space-localized, iterating frequencies. Biology instead, needs time variables, indeed more than one time dimension, in our approach, and they are set by the theory, a priori. Moreover, life's becoming is a plastic tuning of rhythms and frequencies, as ever changing, mathematically heterogeneous, reproduction of geometric and time's forms.

67 See Sarti et al. (2019).

68 See Ronchi (2011).

69 See Soto et al. (2016a). 


\section{Biological Twins vs. Atomic Clocks}

Thought experiments are very important in science. Yet, they must be proposed or understood at the right phenomenal level and possibly not based on nor forcing a philosophical bias. Archimedes imagined a "bag of water" in ... water and proposed his principle. Galilei thought of a falling body in a boat in uniform movement and understood the relativity of movement. Einstein dared to take the point of view of a photon surfing on a light wave. Turing imagined himself as a "human computer" writing 0s and 1s on "a child's arithmetic book", in a perfectly "desultory manner" ${ }^{70}$ As written and as they are commonly interpreted, in reference to the phenomena they refer to, these deep and original insights by imagination are sound and very expressive.

Consider instead the ancient Zeno's paradox about the arrow never reaching a target as it first needs to pass by $1 / n$-th of the distance to the target, for all $n>1$. This is a fantastic mathematical invention, a very early reflection on infinity: the "paradox" of the infinite in the finite or of the infinite divisibility of a continuous segment. It opened the way to Euclid's geometry of continua and, later, to the infinitesimal calculus that made western science. However, it is a physical nonsense. A figure of thought should be compatible with effective observations, as the four founding thought experiments above, in particular with measurement, the only form of quantifiable access we have to (physical) reality. No length of a physical object, no position of a tip of an arrow, may be given by a rational or real number, exactly: classical measurement is always approximated, it is an interval, at least because of thermal fluctuations. A fortiori, if one refers to or measures the position of a moving arrow, one always obtains an interval, both in space and time. That is, the arrowhead is in an interval of space and a moving one will be even more grossly approximated, in time, as time measurement yields always a durée. And the physical "paradox" vanishes.

Bergson, since the 1880's, stressed that the Zeno's mathematical invention is based on a lack of understanding of physical movement. ${ }^{71}$ Bergson criticizes the implicit identification of space and time and the missing appreciation of the durée, which is proper, in particular, to the understanding of movement. ${ }^{72}$ And

70 See Longo (2018c).

71 See Bergson (1910).

72 "More generally, in that continuity of becoming which is reality itself, the present moment is constituted by the quasi-instantaneous section effected by our perception in the flowing mass; and this section is precisely that which we call the material world" (Bergson 1947, 178). 
he was right: the paradox is physically meaningless, while being one of the founding remarks of western mathematics. ${ }^{73}$

Similarly, Bergson is not at ease with the "twins' paradox" as it was later called Langevin's example of relativistic delays of clocks under different accelerations, described as differently aging humans. Unfortunately, Bergson tries to criticize the paradox in physical terms, by claiming that each twin equivalently (symmetrically) moves w.r.to the other - this is wrong since one is in an inertial system (sitting on Earth), while the other is accelerated. However, Bergson's critique is also based on his understanding of the time of consciousness as a tissue of interacting durées. From our perspectival epistemology, his view of time as a dialogue of consciousness is beyond biology and its proper scientific objectivization. Moreover, Bergson searches for a universal time, if not an absolute, by referring to an identity of intimate durées in subjectivistic terms. ${ }^{74}$ However psy-

73 For the reader who considers this argument too "physicalist" or even simplistic, more philosophical insights may be found in Ronchi (2011); Miquel (2013); During (2014). I entirely share, in particular, Ronchi's Bergsonian critique of the identification of space and time and his analysis of Bergson's and other philosophers' deep reflections, since Greek philosophy, on continua and movement, also inspired by Zeno's paradox. However, some ambiguities do not allow to focus on properly mathematical and physical theorizing on these matters. For example, Bergson claims that one can always divide une chose (matter? an object?), but not an action. Also, a chose though cannot be divided indefinitely, nor can space or time, both in a classical and in a quantum physical understanding of "dividing" -only pure mathematics allows it, in particular in the Cantorian Universe of Sets, a piling up of dimensionless points. More recent Topos Theoretic approaches, since Grothendieck (Verdier et al. 1972), may provide a better fit with the relativizing objectivity of physics, beyond the Absolute and Stratified Universe of Sets and Points still prevailing in the mathematics and philosophy of physics. Diverse Universes (categories and sheaves), "with no points" nor stratified (not "predicative") in Johnstone (1977); Asperti and Longo (1991); and their relative transformations may allow to revisit the debate in physics and philosophy (see Zalamea 2012; Longo 2015). In particular, Categories and Toposes provide an analysis of invariant concepts in mathematics methodologically much closer to Einstein's Invariantentheorie, as he preferred to call his own theory, than the Cantorian-Russellian stratified absolutes still prevailing, even in physics. These absolutes provide an image of or a mathematical projection on nature of a foundation built on point-elements as solid bricks on top of solid bricks, a parody of complexity as the stacking of the simple. A recent category-theoretic approach, where Lagrangian submanifolds in symplectic geometry form the indivisible, but complex elements, proposes interesting bridges between relativistic gauge and quantum indetermination (Catren 2014). We did and may invent more mathematics beyond the fantastic one which leads from Zeno to Cantor.

74 In Bergson's long argument, Pierre and Paul measuring relativistic time in S and S', live the same durée: "Hence, the time lived and recorded in the system, the time inside of and immanent in the system, in short, real time, is the same for S and S"' (Bergson 1965, 71). Thus, la durée is the same for the two conscious beings and only by mathematics one may abstractly understand the different speed of the clock of the other, that is that they are in differently accelerated refer- 
chological, his views are grounded on living organisms, our reference here, with their own rhythms, as internal clocks, and their durées. Let's then develop our focus on biological rhythms, which allow to measure aging. As we noticed, they are a condition of possibility for Bergson's durée in consciousness.

The "paradox" of time measurements in different reference systems is a fantastic physical insight since the early days of RT and empirically corroborated by astronomical measurements and human made atomic clocks - the time of GRT applies to the clocks on our satellites (GPS uses it). Yet, it is biologically misleading. Biological rhythms are either resilient to differences in nano-seconds or it makes little physical and biological sense to imagine a viable ecosystem sufficiently accelerated as to be taken close enough to the speed of light and obtain biologically relevant time differences w.r.to an inertial one. As for the first issue, quantum phenomena and their timing may be relevant in biology as they may have phenotypic effects, ${ }^{75}$ but nano-seconds are irrelevant in relating organisms' biological rhythms. ${ }^{76}$ They are as irrelevant as measuring micro-fluctuations in meteorology or as integrating Schrödinger's equation for the quanta composing water in the hydrodynamic analysis of the El Nino oceanic current, a dynamics of incompressible fluids better understood in continua. ${ }^{77}$ Secondly, no complex organism can stand much more than $1 \mathrm{~g}$ acceleration beyond a short time lapse. Then, what kind of viable ecosystem and how much energy is it needed to accelerate such an ecosystem at about $1 \mathrm{~g}$ for years, once away from Earth? Energetic considerations and the tissue of correlations of biological rhythms and frequencies, required for life, are out of the scope of this theatrical thought experiment. Bergson may be technically wrong, but he is sending us a warning not to conflate the two dimensions of time (rhythms vs. frequencies), similarly as he had suggested not to confuse mathematics and physical movement in Zeno's paradox. ${ }^{78}$

ence systems: "What is it then, if not a mere mathematical expression meant to indicate that Peter's not Paul's system has been taken as the system of reference?” (Bergson 1965, 72). Bergson, while appreciating Einstein's Invariantentheorie, warns against giving an ontological status to mathematical invariants.

75 See Buiatti and Longo (2013) for examples.

76 In photosynthesis, picoseconds may matter as for energy transfer. But this fundamental interface inert/life is far from biological rhythms.

77 See Chibbaro et al. (2015).

78 Montévil and a biologist and physician friend, a collaborator of the European Space Agency (ESA), suggested that "it is the traveling twin that will get old sooner! Whatever we do to reconstruct an artificial ecosystem in a spaceship, by the time (his biological rhythms) required for the experiment at $1 \mathrm{~g}$ acceleration (an animal cannot stand more, nor less, for long), the bio-psychic stress will heavily affect his health, thus aging and life expectancy". By the periodic experience of much less isolated and stressing environments, merchant ships, navigators happen to have 
In summary, the choice of the right scale of access and measurement as well as the analysis of the pertinent observables and interactions, are at the core of the scientific investigation. After 1945, Einstein went back to the issue of time: "what about the psychological origin of the concept of time?" (Einstein 1954, 79). His answer refers to different observers comparing lightnings that would appear in different order of time according to different distances, in view of the bounded speed of light: "In order to arrive at the idea of an objective world, an additional constructive concept still is necessary: the event is localised not only in time, but also in space" (Einstein 1954, 79). A very pertinent argument in astronomy or for timing by atomic human made clocks, but a biologically irrelevant issue as for interacting organisms in their niches and ecosystems on Earth. Jokes are fun (drolligsten) also said Einstein in reference to the twins' paradox, but they must be limited at their scale of pertinence - so, in his late years, Einstein often and more openly reconsidered Bergson's arguments. ${ }^{79}$

Thus, in our view, Bergson disagreed with the abuses of many physicists who considered and still now consider a quantum or a relativistic theory of time as the theory to which all other theories should be reduced or even as a theoretical absolute - this theory "is a metaphysic grafted upon science, it is not science” (Bergson 1965, 63). Note finally, that, without following Bergson's universalistic-metaphysical argument, organisms on Earth do have a universal or global clock, coordinating also their autonomous rhythms, their durées: the spinning of Earth on itself, its movement around the Sun, the turning Moon, three fundamental physical frequencies beating the "a priori" background time of life. ${ }^{80}$

five years shorter life expectancy (https://syndicoop.info/marine-marchande-un-constat-alarm ant/). The physicist may observe that he/she does not deal with these issues. Then, he/she should better compare only physical clocks.

79 See Canales (2015), Ch. 28-29.

80 As observed above, Bergson, while rejecting Newton's absolute time, focuses on the unity of material time within a universe characterized by a plurality of rhythms of duration (durée), but also on the idea of an absolute of movement captured in durée, irreducible to the reference framework imposed by the principle of the relativity of movement, as stressed in During 2014 , 7. This would provide a global or universal time-framework, as a principle of coordination and homogenization of flows of heterogeneous durées (During 2014, 7; Bergson 1965, 47), which is our approach as for life, on this Earth. 


\section{Conclusion}

Our critique joins Bergson's not because of some physicists' lack of attention to the evidence of time, since science is a constant fight against evidence and common sense, but because we reject a view of science as the occupation of reality by already mastered tools, with little theoretical care for the specificities of different phenomenal domains. And this, against the extraordinary history of inventiveness which is proper to physics. Biology instead, the most difficult scientific discipline as Einstein wrote in a letter to Schrödinger, should be grounded just on some physics of macromolecules - plus some vague references ("metaphores") to DNA centred information and programming with heavy consequences on research. ${ }^{81}$

We must acknowledge though that our objectivizing interpretation of Bergson's durées is not straightforward, as it is instrumentally forced in order to discuss today's approaches to biological time. Poincaré (1917), soundly describes it differently, that is, in his views, the conception of time in Bergson is that durée which, far from being a pure quantity free of any quality, is the very quality itself whose various parts partly penetrate each other and differ qualitatively from each other. For Poincaré, this durée could not be an instrument for scientists; it could only play this role by undergoing a profound transformation, by spatializing itself, as Bergson says. It had to become measurable, since what cannot be measured cannot be an object of science. Now, measurable time is also essentially relative. Moreover, psychological time, the Bergsonian durée, from which the scientist's time has come out, serves to classify the phenomena that occur in the same consciousness. Poincare concludes by observing that it is powerless to classify two psychological phenomena that have two different consciences as their theater or, a fortiori, two physical phenomena.

We tried here to set the notion of durée at the core of a tissue of objectiverelative correlations in biology, as a science, following Relativity's main epistemic teaching, yet without identifying/subordinating time to space. In this attempt, we did not need to assume a universal consciousness nor to attribute "consciousness" to all forms of life (consciousness is coextensive to life for Bergson). The changing evolutionary and historical nature of "consciousness" must instead be acknowledged: this notion requires an analysis of the "critical transitions" that may help to single out its constitution. For example, the invention of human language and of ... writing, which allowed to see the invisible, language and our own thinking, to "reflect" on them, are two of these most recent transi-

81 See Longo (2018b). 
tions. Then the western consciousness of time, from the vision of a circle, an iteration with no novelty in ancient Greece, in Pytagora's school and in the tragic iteration of events in Aeschylus, became an open-ended line from Sophocles to Kant. ${ }^{82}$ A uniform or universal conception of consciousness, of time in particular, for all living entities, independent from biological and human history, is beyond our scientific perspective.

However, does a scientific concept strictly need to be "measurable”? Joining Poincaré, both Bergson and Einstein would say so. Thus, Bergson's principial denial of the scientific nature and measurability of notions such as time durée and randomness justifies Poincaré's critique and drives Bergson's analysis towards a metaphysics away from biology, as a science. Metaphysical investigations are very interesting, but are a different, highly needed, framing job -if the molecular biologists of the Central Dogma were at least conscious of their metaphysics, we would perhaps have some better genetics, from GMOs to cancer research. ${ }^{83}$

In our scientific attempt, we first tried to specify durées by biological rhythms and characteristic times and, thus, suggested possible tools to measure them. Moreover, we singled out and qualified with enough, we hope, scientific rigor the notion of historical time and of biological randomness - as relative unpredictability (see the references more than the cursory presentation in $\S 3$ ). In our approach, as we pose the dynamics of the very phase spaces and stress the role of rare events, evolutionary randomness is not measurable, by probabilities typically. Science though may also proceed by first singling out conceptual contours and qualifications at the interface with phenomena, then, perhaps, but not necessarily, provide new mathematical spaces for measurement. These spaces, yet to be invented (but Hilbert spaces for QM were also a late invention), may be a forthcoming development of the work in (Sarti et al 2019), as for historicity and randomness.

Finally, the constructed objectivity of time proposed here, with its different roles (regulating vs. constitutive, in physics vs. biology), does not exclude the knowing subject. There is always a residual of it in the choice of the reference system and its metrics, such as the choice of the coarse graining, as for time, as well as in defining the transformations that single-out the invariants w.r. to that choice. On the one side, though, a living organism does objectively fix the thermal time coarse graining, as we observed. On the other, we decided to work at the organismal and evolutionary levels, as for their timing: the theoretical choice is perspectival and its empirical grounding requires fixing also ob-

82 See Redondi (2007); Deleuze (1978).

83 See Longo (2018b). 
servables and measurements, as Einstein observed. Thus, a philosophical reflection on consciousness of time, à la Bergson, may help to historicize our perspectival construction: our own open ended, creative appreciation of the biological time operator is the result of a historical formation of sense. In order to set it on sufficiently robust grounds, we tried to raise the epistemological question of its scientific pertinence by an analysis of the

“... oppositional pair: subjective-absolute and objective-relative [that] seems ... to contain one of the most fundamental epistemological insights that can be extracted from natural sciences [...] But perhaps this question can be answered by pointing toward the essentially historical nature of that life of the mind of which my own existence is an integral but not autonomous part. It is light and darkness, contingency and necessity, bondage and freedom, and it cannot be expected that a symbolic construction of the world in some final form can ever be detached from it” (Weyl 1949, 62).

\section{References}

Amsterdamski, Stefan/Pomian, Krzysztof (eds.) (1990), La querelle du déterminisme, Paris.

Arani, Raffaella/Bono, Ivan/Del Giudice, Emilio/\& Preparata, Giuliano (1995), “QED coherence and the thermodynamics of water", in : International Journal Physics B9, 1813-1841.

Aspect, Alain/Grangier, Philippe/Roger, Gérard (1982), “Experimental Realization of the Einstein-Podolsky-Rosen-Bohm Gedankenexperiment: A New Violation of Bell's Inequalities", in: Physical Review Letter 49, 91-94.

Asperti, Andrea/ Longo, Giuseppe (1991), Categories, Types and Structures, New York.

Bailly, Francis/Longo, Giuseppe (2009), “Biological organization and anti-entropy”, in: Journal of Biological Systems 17 (1), 63-96.

Bailly, Francis/Longo, Giuseppe (2011), Mathematics and the natural sciences: the physical singularity of life, London (original French version: Paris 2006).

Bailly, Francis/Longo, Giuseppe/Montévil, Maël (2011), “A 2-dimensional Geometry for Biological Time”, in: Progress in Biophysics and Molecular Biology 106 (3), 474-84.

Bergson, Henri (1910), Time and Free Will: An Essay on the Immediate Data of Consciousness, London.

Bergson, Henri (1922), Creative Evolution, London.

Bergson, Henri (1946), The Creative Mind, New York.

Bergson, Henri (1947), Matter and Memory, New York.

Bergson, Henri (1965), Duration and Simultaneity: with Reference to Einstein's Theory, New York.

Berthoz, Alain (2000), The Brain Sens of Movement, Cambridge, MA.

Binney, James et al. (1992), The Theory of Critical Phenomena: An Introduction to the Renormalization Group, New York.

Bouton, Christophe/Huneman, Philippe (2018), Temps de la nature, nature du temps, Paris (original version in English: Berlin 2015).

Callender Craig (2017), What makes time special, Oxford. 
Calude, Christian S./Longo, Giuseppe (2016), “Classical, Quantum and Biological Randomness as Relative Unpredictability”, Special issue of Natural Computing 15 (2), $263-278$.

Canales, Jimena (2015), The Physicist and the Philosopher, Princeton, New Jersey.

Catren, Gabriel (2014), “On the Relation Between Gauge and Phase Symmetries”, in: Foundations of Physics 44, 1317-1335.

Chibbaro, Sergio/Rondoni, Lamberto/Vulpiani, Angelo (2015), Reductionism, Emergence and Levels of Reality: The Importance of Being Borderline, Berlin.

Chirco, Goffredo/Josset, Thibaut, Rovelli, Carlo (2016), "Statistical mechanics of reparametrization-invariant systems. It takes three to tango", in: Classical and Quantum Gravity 33 (4), 1-9.

Connes, Alain (1994), Non-commutative Geometry, New York.

Connes, Alain/Rovelli Carlo (1994), "Von Neumann algebra automorphisms and time-thermodynamics relation in general covariant quantum theories", in: Classical and Quantum Gravity 11-12, 2899-2918.

Del Giudice, Emilio et al. (1983), "Spontaneous symmetry breakdown and boson condensation in biology", in: Physics Letters A 95 (9), 508-510.

Del Giudice, Emilio et al. (1986), “Electromagnetic field and spontaneous symmetry breakdown in biological matter”, in: Nuclear Physics B 275 (2), 185-199

Deleuze, Gilles (1978), Deuxième leçon sur Kant (21/3/1978), in: http//:le-terrier.net

Disertori, Margherita/Sabot Christoph/Tarrès Pierre (2015), "Transience of Edge-Reinforced Random Walk", in: Communications in Mathematical Physics 339 (1),121-148.

Dorato, Mauro (2016), “Rovelli’s Relational Quantum Mechanics, Anti-Monism, and Quantum Becoming”, in: David Yates/Anna Marmodoro (eds.) The Metaphysics of Relations, Oxford.

During, Elie (2009), Introduction au dossier critique de Henri Bergson : Durée et simultanéité, Paris, $219-244$.

During, Elie (2014), “Langevin ou le paradoxe introuvable”, in : Revue de Métaphysique et de Morale 4, 513-527.

Edelman, Gerald M./Gally, Joseph A. (2001), “Degeneracy and Complexity in biological systems", in: Proceedings of the National Academy of Science 24, 13763-13768.

Einstein, Albert (1954), Relativity and the Problem of Space, http://www.relativitybook.com/re sources/Einstein_space.html

Einstein, Albert/Podolsky, Boris/Rosen, Nathan (1935), “Can Quantum-Mechanical Description of Physical Reality be Considered Complete?”, in Physical Review 41, 696-702.

Feynman, Richard (1964), The Feynman lectures on Physics, vol. 1, 46-7 in: FLP Vol. I Table of Contents (caltech.edu)

Foster, Russell G./Kreitzman, Leon (2004), Rhythms of life. The Biological Clocks that Control the Daily Lives of Every Living Thing, London.

Frisch, Mathias/Pietsch, Wolfgang (2016), "Reassessing the Ritz-Einstein debate on the radiation asymmetry in classical electrodynamics", in: Studies in History and Philosophy of Modern Physics 55, 13-23.

Gayon, Jean/Montévil, Maël (2017), “Repetition and Reversibility in Evolution: Theoretical Population Genetics”, in: Christophe Bouton/Philippe Huneman (eds.), Time in Nature and the Nature of Time, Berlin, 275-314. 
Gould, Stephen J. (1966), “Allometry and Size in Ontogeny and Phylogeny”, in: Biological Review of the Cambridge Philosophical Society 41, 587-640.

Gould, Stephen J. (1996), Full House, New York.

Gould, Stephen J. (2002), The Structure of evolutionary Theory, Cambridge, MA.

Günther, Bruno/Morgado, Enrique (2005), "Allometric scaling of biological rhythms in mammals", in: Biological Research 38, 207-212.

Hayflick, Leonard (2007), "Entropy explains aging, genetic determinism explains longevity, and undefined terminology explains misunderstanding both”, in: PLoS Genet 3 (12), 2351- 2354.

Huneman, Philippe (2018), “Macroévolution et microévolution. Le problème de l'échelle de temps dans la biologie évolutive”, in : Christophe Bouton/Philippe Huneman (eds.), Time in Nature and the Nature of Time, Berlin, 343-370.

Johnstone, Peter T. (1977), Topos Theory, London.

Koppl, Roger/Kauffman, Stuart/Longo, Giuseppe/Felin, Teppo (2015), “Economy for a Creative World", in: Journal of Institutional Economics 11 (1), 1-31.

Kosman-Schwarback, Yvette (2010), The Noether theorems: Invariance and Conservation Laws in the Twentieth Century, Berlin.

Lecointre, Guillaume/Le Guyader, Hervé (2017), Classification phylogénétique du vivant, quatrième édition (tome 2), Paris.

Lesne, Annick (2018), "Les échelles de temps dans les systèmes naturels et leur modélisation”, in: Christophe Bouton/Philippe Huneman (eds.), Time In Nature And The Nature Of Time, Berlin, 67-84.

Longo, Giuseppe/Montévil, Maël (2011a), "Protention and retention in biological systems", in: Theory in Biosciences 130 (2), 107-117.

Longo, Giuseppe/Montévil, Maël (2011b), “From physics to biology by extending criticality and symmetry breakings”, in: Progress in Biophysics and Molecular Biology 106 (2), $77-91$.

Longo, Giuseppe/Montévil, Maël (2012), "Randomness Increases Order in Biological Evolution”, in: Micheal Dinneen/Bakhadyr Khoussainov/André Nies (eds.), Computation, Physics and Beyond, Berlin/Heidelberg, 289-308.

Longo, Giuseppe/Montévil, Maël/Kauffman, Stuart (2012), “No entailing laws, but enablement in the evolution of the biosphere", in: ACM proceedings of Genetic and evolutionary Computation Conference, GECCO'12, Philadelphia (PA, USA), July 7-11.

Longo, Giuseppe/Montévil, Maël (2014), Perspectives on Organisms: Biological Time, Symmetries and Singularities, Dordrecht.

Longo, Giuseppe (2015), Conceptual Analyses from a Grothendieckian Perspective: Reflections on Synthetic Philosophy of Contemporary Mathematics by Fernando Zalamea, in: Speculations IV, https://punctumbooks.com/titles/speculations-vi/.

Longo, Giuseppe (2016), A review-essay on reductionism: some reasons for reading, in: Chibbaro, Sergio/Rondoni, Lamberto/ Vulpiani, Angelo, Reductionism, Emergence and Levels of Reality. The Importance of Being Borderline, London, https://www.urbanomic. com/document/on-the-borderline/

Longo, Giuseppe (2017), "How Future Depends on Past Histories and Rare Events in Systems of Life", in: Foundations of Science 23, 443-474. 
Longo, Giuseppe/Montévil, Maël (2017), “Comparing Symmetries in Models and Simulations”, in: Lorenzo Magnani/Tommaso Bertelotti (eds.), Handbook of Model-Based Science, Berlin, 843-856.

Longo, Giuseppe (2018a), “Interfaces of Incompleteness”, in: Gianfranco Minati/Mario R. Abram/Eliano Pessa (eds.), Systemics of Incompleteness and Quasi-systems, New York, $3-55$.

Longo, Giuseppe (2018b), “Information and Causality: Mathematical Reflections on Cancer Biology”, in: Organisms. Journal of Biological Sciences 2 (1), 83-103.

Longo, Giuseppe (2018c), “Letter to Alan Turing”, in: Füller, Matthew/Braidotti, Rosi (eds), Theory, Culture and Society, Special Issue on Transversal Posthumanities 85-101.

Longo, Giuseppe (2020), “Naturalizing Physics. Or, embedding physics in the historicity and materiality of the living", in: Deleuziana $11,1-15$.

Longo, Giuseppe/Longo, Sara (2020), "Infinity of God and Space of Men in Painting, Conditions of Possibility for the Scientific Revolution”, in: Ruth Scheps/Marie-Christine Maurel (eds.), Mathematics in the Visual Arts, London, 1-28.

Maestrini, Davide. et al (2018), “Aging in a Relativistic Biological Space-Time”, in: Frontiers in Cell and Developmental Biology 6, 1-23.

McTaggart, John E. (1908), “The Unreality of Time”, in: Mind 17 (68), 457-474.

Memmott, Jane/Craze, Paul G./Waser, Nickolas M./Price, Mary V. (2007), “Global warming and the disruption of plant-pollinator interactions", in: Ecology Letters 10(8), 710-717.

Miquel, Paul-Antoine (2013), Bergson dans le miroir des sciences, Paris.

Miquel, Paul-Antoine (2015), Sur le concept de Nature, Paris.

Montévil, Maël / Mossio, Matteo (2015), "Closure of constraints in biological organization”, in: Journal of Theoretical Biology 372, 179-191.

Montévil, Maël (2019), “Measurement in biology is methodized by theory”, in: Biology \& Philosophy 34, 35-50.

Montévil, Maël (2020a), “De l'œuvre de Turing aux défis contemporains pour la compréhension mathématique du vivant", in : Intellectica (submitted).

Montévil, Maël (2020b), "Entropies and the Anthropocene crisis”, in: Al and Society, (submitted).

Mugur-Schachter, Mioara/Longo, Giuseppe (eds.) (2014), Developments of the Concepts of Randomness, Statistic, and Probability, Special issue of Mathematical Structures in Computer Science24 (3).

Nicolis, Gregoire/Prigogine, Ilya (1977), Self-organization in non-equilibrium systems, New York.

Noble, Denis (2006), The Music of Life, New York.

Noble, Denis (2012), “A theory of biological relativity: no privileged level of causation”, in: Interface Focus 2 (1), 55-64.

Paaby, Annalise B./Rockmann Matthew (2014), “Cryptic genetic variation: evolution's hidden substrate", in: Nature Reviews Genetics 15 (4), 247-258.

Pavesi, Angelo/Vianelli, Alberto/Chirico, Nicola et al. (2018), "Overlapping genes and the proteins they encode differ significantly in their sequence composition from non-overlapping genes", in: PLOS ONE 13(10): e0202513.

Perret, Nicole/Longo, Giuseppe (2016),"Reductionist perspectives and the notion of information", in: Ana Soto/Giuseppe Longo/Denis Noble (eds.) (2016), “From the century 
of the genome to the century of the organism: New theoretical approaches", a Special issue of Progress in Biophysics and Molecular. Biology 122 (1).

Peters, Robert H. (1983), The ecological implications of body size, New York.

Poincaré, Jules H. (1917), Dernières pensées, Paris.

Raff, Rudolf A./Wray, Gregory A. (1989), “Heterochrony: developmental mechanisms and evolutionary results”, in: Journal of Evolutionary Biology 2 (6), 409-434.

Redondi, Pietro (2007), Storie del tempo, Roma-Bari.

Ritz, Walter/Einstein, Albert (1909), “Zum gegenwärtigen Stand des Strahlungsproblems”, in: Physikalische Zeitschrift 10 (9), 323-324.

Ronchi, Rocco/Leoni, Federico (2007), Metafisica e psicologia in Bergson, in: Bergson, Henri, Storia della memoria e storia della metafisica, Pisa, 7-42.

Ronchi, Rocco (2011), Bergson. Una sintesi, Milan.

Rovelli, Carlo (1993), "Statistical mechanics of gravity and the thermodynamical origin of time”, in: Classical and Quantum Gravity 10, 1549-1566.

Rovelli, Carlo (2011), "Forget time: Essay written for the FQXi contest on the Nature of Time", in: Foundations of Physics 41 (9), 1475-1490.

Rovelli, Carlo (2015), “Is Time’s Arrow Perspectival?”, in: ArXiv: https://arxiv.org/abs/1505. 01125

Rovelli, Carlo (2018a), The order of Time, New York.

Rovelli, Carlo (2018b), "Space and Time in Loop Quantum Gravity”, in: ArXiv: https://arxiv. org/abs/1802.02382

Rovelli, Carlo/Vidotto Francesca (2018), “Pre-big-bang black-hole remnants and past low entropy”, in: Universe 4 (129), https://www.usb.ac.ir/FileStaff/7194_ $2018-11-18-20-48-48 . p d f$

Saigusa, Tetsu/Tero, Atsushi/Nakagaki, Toshiyuki/Kuramoto Yoshiki (2008), “Amoebae Anticipate Periodic Events”, in: Phyiscal Review Letters, 100 (1), 018101.

Sarti, Alessandro/Citti, Giovanna/Piotrowski, David (2019), "Differential heterogenesis and the emergence of semiotic function", in: Semiotica 230, 1-34.

Sethna, James P. (2006), Statistical mechanics: Entropy, order parameters, and complexity. New York.

Shlesinger, Tom/Loya, Yossi (2019), "Breakdown in spawning synchrony: A silent threat to coral persistence”, in: Science 365 (6457), 1002-1007.

Soto, Ana/Longo, Giuseppe/Noble, Denis (eds.) (2016), “From the century of the genome to the century of the organism: New theoretical approaches", a Special issue of Progress in Biophysics and Molecular. Biology 122 (1).

Soto, Ana/Longo, Giuseppe/Montévil, Maël/Sonnenschein, Carlos (2016a), “The biological default state of cell proliferation with variation and motility, a fundamental principle for a theory of organisms", in: Progress in Biophysics and Molecular Biology 122 (1), $16-23$.

Thom, René (1980), “Halte au hasard, silence au bruit”, in: Le Débat 3 (3), 119-132.

Uzan, Jean-Philippe (2011), "Varying Constants, Gravitation and Cosmology”, in: Living Reviews in Relativity 14 (2) 118-155.

Van Fraassen, Bas (1985), An introduction to the philosophy of time and space, New York. Verdier, Jean-Louis/Saint-Donat, Bernard/Grothendieck, Alexandre (1972), Théorie des Topos et Cohomologie Etale des Schémas, (2 vols.) Berlin/Heidelberg. 
Vulpiani, Angelo/Cecconi, Fabio/Cencini, Massimo/Puglisi, Andrea/Vergni, Davide (eds.)

(2014), Large Deviations in Physics, Berlin.

Wesson, Paul S. (1999), Space-Time-Matter, Modern Kaluza-Klein Theory, Singapore.

West-Eberhard, Mary J. (2003), Developmental Plasticity and evolution, New York.

West-Eberhard, Mary J. (2005), "Developmental plasticity and the origin of species

differences", in: PNAS 102 (1), 6543-6549.

Weyl, Hermann (1919), Raum, Zeit, Materie, Berlin.

Weyl, Hermann (1949), Philosophy of Mathematics and of Natural Sciences, Princeton,

Princeton, New Jersey.

Weyl, Hermann (1952), Symmetry, Princeton, New Jersey.

Williams Kathy S./Simon, Chris (1995), "The Ecology, Behavior, and evolution of Periodical Cicadas", in: Annual Review of Entomology 40, 269-295.

Yang, Chen-Ning/Mills, Robert M., 1954, "Conservation of isotopic spin and isotopic gauge invariance”, in: Physical Review 96 (1), 191-196.

Zalamea, Fernando (2012), Synthetic Philosophy of Contemporary Mathematics, Cambridge, MA. 
